\title{
Long-term transients and complex dynamics of a stage- structured population with time delay and the Allee effect.
}

\author{
A. Yu. Morozov ${ }^{1}$, M. Banerjee ${ }^{2 *}$, S. V. Petrovskii ${ }^{1}$ \\ ${ }^{1}$ Department of Mathematics, University of Leicester, LE1 7RH, UK \\ ${ }^{2}$ Department of Mathematics and Statistics, Indian Institute of Technology, Kanpur, INDIA \\ *Corresponding author: M. Banerjee, malayb@iitk.ac.in
}

Keywords: population dynamics, complexity, extinction, chaos, multiple attractors 


\begin{abstract}
Traditionally, mathematical modelling in population ecology is mainly focused on asymptotical behaviour of the model, i.e. as given by the system attractors. Recently, however, transient regimes and especially long-term transients have been recognized as playing a crucial role in the dynamics of ecosystems. In particular, long-term transients are a potential explanation of ecological regime shifts, when an apparently healthy population suddenly collapses and goes extinct. In this paper, we show that the interplay between delay in maturation and a strong Allee effect can result in long-term transients in a single species system. We first derive a simple 'conceptual' model of the population dynamics that incorporates both a strong Allee effect and maturation delay. Unlike much of the previous work, our approach is not empirical since our model is derived from basic principles. We show that the model exhibits a high complexity in its asymptotic dynamics including multi-periodic and chaotic attractors. We then show the existence of long-term transient dynamics in the system, when the population size oscillates for a long time between locally stable stationary states before it eventually settles either at the persistence equilibrium or goes extinct. The parametric space of the model is found to have a complex structure with the basins of attraction corresponding to the persistence and extinction states being of a complicated shape. This impedes the prediction of the eventual fate of the population, as a small variation in the maturation delay or the initial population size can either bring the population to extinction or ensure its persistence.
\end{abstract}




\section{Introduction}

Understanding the aspects and tendencies of complex population dynamics observed in natural ecosystems (Ellner and Turchin, 1995; Ranta et al., 1995; Widdicombe et al., 2010) as well as in the laboratory (Costantino et al., 1997; Yoshida et al., 2003; Becks et al. 2005) is important for both theoretical and practical reasons, and indeed it has been a central point of ecological research for several decades (Nicholson, 1954; Luckinbill, 1974; Turchin, 2003; Odum and Barrett, 2004; Begon et al., 2005). Whilst empirical studies provide invaluable information such as, for instance, examples of different dynamics (Becks et al. 2005; Yoshida et al., 2003), a theoretical approach is necessary in order to generalize and systematize the results of field observations or lab experiments. In particular, mathematical modelling is proved to be an efficient research tool that helps to reveal ecosystem properties when replicated experiments under controlled conditions are not possible (Maynard Smith 1974; Bjornstad and Grenfell, 2001; Turchin, 2003).

Mathematical analysis of population dynamics models usually focuses on asymptotic behavior, considering the existence and stability of steady states, limit cycles or more complex attractors. One reason for this is purely technical: mathematical models describing the system in the large time limit are often much simpler than the 'full' models accounting for transients. In particular, the first stage in a standard 'protocol' to explore the properties of a dynamical system is to reveal its stationary states; the corresponding ODEs become reduced to a system of algebraic equations. Another, more conceptual reason behind the traditional analysis is the assumption (often implicit) that the system will normally stay in the vicinity of an attractor, and hence the steady states' analysis is representative of the system dynamics in general.

However, this traditional way of thinking has recently been challenged. There is a growing understanding that in natural ecosystems the asymptotic behavior is, in fact, rarely seen and their dynamics are essentially transient (Cushing et al., 1998; Hastings 2001; Ims et al., 2008).

Correspondingly, a big new challenge for theoretical ecology is to understand the implications of the transient dynamics (Hastings, 2004). This difficult task is exacerbated by the possibility of long-living transients and "ghost attractors" - when the population dynamics mimic the asymptotic behavior of one kind for a very long time before a sudden fast convergence to a different attractor (Lai and Winslow, 1995; Rodrigues et al. 2011).

Recently, considerable attention has been paid to the identification and prediction of "regime shifts" (Scheffer et al. 2001; Kéfi et al., 2014) - sudden changes that often result in species extinction and biodiversity loss. Understanding the mechanisms and scenarios of such shifts is thought to be important for efficient ecosystem management and nature conservation. There have been many 
reported cases when a species that has been present in apparently safe numbers goes extinct suddenly and seemingly without any warning (Scheffer et al., 2001, 2009; Carpenter, 2003). One general way to explain these cases might be to relate them to catastrophic changes in the ecosystem properties such as, in mathematical terms, the disappearance of a steady state as a result of saddle-node bifurcation (Scheffer et al., 2009, Boettiger and Hastings, 2012). Another scenario of regime shifts can be the existence of long-living transients, which provides an alternative explanation of sudden species extinction (Tilman et al., 1994, Schreiber, 2003). However, specific biological mechanisms and factors resulting in long living transients in various models using different mathematical frameworks often remain obscure (Hastings, 2004). Similarly, it remains unclear how model parameterization can affect its property to exhibit long-living transients as many widely used population dynamics models appear to be structurally unstable with regard to a small change in the constituent model functions (Fussmann and Blasius, 2005; Adamson and Morozov, 2013). In this paper our goal is to show that long-living transients mimicking complex dynamics are likely to be a common property of a conceptual, baseline single species population dynamics model.

The question that immediately arises is what sort of population dynamics model can be regarded as conceptual, but still realistic. Such a model should take into account some common, typical features of real-world populations but describe them in the simplest possible way, avoiding unnecessary details. Arguably, two typical properties of realistic populations are a time delay in reproduction and the Allee effect.

Time delays are widely recognized as an inherent feature of population dynamics (Beretta and Kuang, 1998; Hansen et al., 1998; May, 2001; Murdoch et al., 2003; Ruan, 2006). Triggered by Hutchinson seminal work (Hutchinson, 1948), there have been a large number of theoretical studies concerned with the implications of time delays for populations and communities (Hastings, 1984; Aiello and Freedman, 1990; Murdoch et al., 2003; Ruan, 2009). Time delays are thought to have a destabilizing effect as a sufficiently long delay often turns an otherwise stable positive steady state unstable (Kuang, 1993; May, 2001, Ruan, 2006, 2009), and hence converts steady population densities into population cycles, although the generality of this scenario remains controversial, e.g. see Jankovic and Petrovskii (2014) and Sen et al. (2014).

Interestingly, in spite of the vast existing literature on time delayed population dynamics, some important issues remain poorly studied. Firstly, it remains unclear which modeling frameworks are biologically reasonable and, correspondingly, what should be the baseline model of single-species population dynamics. Earlier studies (Hutchinson, 1948; Aiello and Freedman, 1990; also Ruan 2006 and references therein) were based on extensions of the logistic equation. However, as we will show 
below, this is only relevant if the maturation time is short. Secondly, the limits of dynamical complexity of time delayed dynamics remain unclear. Whilst delay-induced periodic population cycles are well known and well understood, it is still unclear whether a simple single species delayed system is capable to produce more interesting dynamics such as chaos and long-living transients.

The Allee effect, defined as a decrease in the per capita population growth rate at small values of the population density (cf. Dennis 1989), is a common feature of ecological populations as it can result from several different mechanisms (Courchamp et al. 1999; Stephens and Sutherland 1999). It is well known that its inclusion can modify the population dynamics significantly (Courchamp et al. 2008), leading to the emergence of long-living transients in particular (Rodrigues et al. 2011).

However, most of the previous theoretical studies were done using instantaneous non-delayed models, leaving the interplay between the strong Allee effect and the time delay poorly understood (but see Jankovic and Petrovskii, 2014). Recently, a model incorporating both time delay and the Allee effect was considered by Liz and Ruiz-Herrera (2015) and the existence of long living transients for some specific values of delay and initial conditions was observed. However, the generality of their results remained unclear, in particular due to the phenomenon of sensitivity of biological models to parameterizations (Cordoleani et al., 2011).

In this paper, we show that the interplay between time lag and the Allee effect can result in complex dynamics and long-term oscillatory transient regimes in a single-species continuous-time population model. Depending on the parameter values and the initial conditions, the population size can exhibit oscillations around the unstable equilibrium keeping constant amplitude for a long time until it eventually settles to one of the two steady states of the system: extinction or persistence. In the context of real-world ecosystems, this means that a population showing apparently 'healthy' dynamics over years of observation can suddenly collapse and go extinct even in a perfectly stationary environment. This is in good agreement with previous studies (cf. Schreiber, 2003; Liz and RuizHerrera, 2015). However, we also show that the parametric space of the system has a complicated structure, and the basins of attraction corresponding to persistence and extinction have complicated shapes, so that small variation of the time delay or the initial conditions can turn extinction to persistence and vice versa. Moreover, we show that the simple time-continuous single species system with the time delay and the Allee effect can exhibit long-living chaotic transients. Along with the transient dynamics we also investigate the long term asymptotic behaviour of the model and show that it can exhibit a wide array of complex dynamics including multiple cycles, intermittent dynamic and chaos. 
We also mention here that the population dynamics model that we use in this study is not empirical; instead, it is derived from certain first principles of the population dynamics. Contrary to the over-simplified Hutchinson model, it takes into account the existence of different life stages such as juveniles and adults, the rate of transition between the stages being affected by the maturation lag. Therefore, in our model the time delay appears naturally as a result of a careful description of the population, rather than being "plugged in" based on purely heuristic arguments, as is often done in the literature (see Sen et al., 2014 for some examples).

The paper is organized as follows. In Section 2, we introduce our conceptual population dynamics model. In Section 3 we study the properties of the model. Firstly, we fulfil a complete bifurcation analysis of the system's attractors (Section 3.1). Then, as adequate mathematical tools for analyzing transients are currently under-developed, we perform an exhaustive study of transients by extensive numerical simulations (Section 3.2). Finally, Section 4 provides a discussion of our findings and concluding remarks. 


\section{Model equations}

We use the general framework of age-structured populations by Gurney et al. (1983). Let us consider a single-species population with continuous reproduction, i.e. new offspring can appear at any time $t$. In order to take into account the effect of maturation, we develop a conceptual continuous-time model of a stage-structured population that consists of juveniles and adults. Adults are capable of reproduction and juveniles are not. Juveniles become adults after a maturation time $\tau$.

Let us denote the densities of juveniles and adults at time $t$ as $x(t)$ and $y(t)$, respectively. The rate of replenishment of the juveniles due to the reproduction of adults at time $t$ (denoted by [ $d x / d t]^{+}$ because it only takes into account replenishment, and not mortality of juveniles) is:

$$
\left[\frac{d x(t)}{d t}\right]^{+}=r y(t),
$$

where $r$ is the per capita reproduction rate that, in a general case, depends on the population density of adults (e.g. due to effects of competition). Correspondingly, the increase $\Delta x(t)$ in the density of juveniles because of the reproduction of the adults occurring over a small interval of time $\Delta t$ is given by the following equation:

$$
\Delta x(t) \approx\left[\frac{d x(t)}{d t}\right]^{+} \Delta t=r y(t) \Delta t .
$$

Let us now consider the change $\Delta y(t)$ in the population density of adults that occurs over the time between $t$ and $t+\Delta t$. Obviously,

$$
\Delta y(t)=\Delta y^{+}(t)-\Delta y^{-}(t),
$$

where $\Delta y^{+}(t)$ is the increase in the density of adults due to the maturation of the juveniles and $\Delta y^{-}(t)$ is the decrease in the density of adults due to their mortality. We assume that the adults mortality is density independent with mortality rate $D$, so that

$$
\Delta y^{-}(t)=D y(t) \Delta t \text {. }
$$

An increase in the density of adults occurs because of the maturation of juveniles. In the course of maturation, juveniles die with mortality rate $\delta$. From the individuals $\Delta x(t)$ that are born between $t$ and $t+\Delta t$, only a certain fraction survives and reaches the state of maturity:

$$
\Delta x^{+}(t+\tau)=\Delta x(t) e^{-\delta \tau},
$$

or, similarly,

$$
\Delta x^{+}(t)=\Delta x(t-\tau) e^{-\delta \tau} .
$$

Those who survive will leave the class of juveniles and enter the class of adults; therefore

$$
\Delta y^{+}(t)=\Delta x^{+}(t) \text {. }
$$

From Eqs.(3-7), we obtain:

$$
\Delta y(t)=\Delta x^{+}(t)-D y(t) \Delta t=\Delta x(t-\tau) e^{-\delta \tau}-D y(t) \Delta t .
$$


Taking now into account (2), Eq. (7) takes the following form:

$$
\Delta y(t)=r y(t-\tau) e^{-\delta \tau} \Delta t-D y(t) \Delta t .
$$

Recall that we have assumed $\Delta t$ to be small, therefore $\Delta y / \Delta t \approx d y / d t$. From (7)-(8), we obtain the following delayed differential equation describing the population dynamics of the stage-structured population:

$$
\frac{d y(t)}{d t}=r e^{-\delta \tau} y(t-\tau)-D y(t) .
$$

Note that Eq.(9) does not contain the density $x(t)$ of juveniles. Therefore, although a full description of the structured population dynamics should also include the equation for $x(t)$ (e.g. see Aiello and Freedman 1990), the main properties of the system can be revealed based on the single equation (9). Once the solution of (9) is known, the density of juveniles can be obtained from Eq.(1). It is worth mentioning here that in the special case where the maturation time is negligible, i.e. $\tau=0$, Eq.(9) coincides with the standard single species model of unstructured population dynamics.

The last issue to decide is the type of the density dependence in the per capita reproduction rate, i.e. the parametrization of function $r(y)$. A generic property of the population dynamics is that, for sufficiently large values of the population density $y$, the per capita reproduction rate $r$ should be a decreasing function of $y$ as a result of the competition for resources and/or space (e.g. Begon et al., 2005). Furthermore, we recall that, for the reasons explained in the introduction, we want our model to take into account the Allee effect. The defining property of the Allee effect is that the per capita reproduction rate reaches its maximum value at a positive value of the population density (i.e. not for $y \rightarrow+0$ as it happens in the case of logistic growth). Therefore, for the purposes of this study, function $r(y)$ should have a local maximum, which means that the graph of the function is dome-shaped.

There are numerous mathematical parameterizations of the per capita reproduction rate $r(y)$ that satisfy the above requirements, e.g. see Boukal and Berec (2002). In order to make our results comparable with some other relevant studies, we consider function $\mathrm{y}(\mathrm{r})$ as follows:

$$
r(y)=\frac{\alpha y^{n-1}}{1+\beta y^{m}}, n<m,
$$

where $\alpha, \beta, n$ and $m$ are positive parameters. Function (10) is therefore an immediate generalization of the parameterizations earlier used by Jacobs (1984) and Takeuchi (1996). Here the coefficient $\alpha$ characterises the reproduction rate at low population level and the coefficient $\beta$ characterises the strength of interspecific competition. The exponents $n$ and $m$ quantify the strength of the density dependence at small and large population density, respectively; the condition $m>n$ is required to ensure that the reproduction rate vanishes at large $y$. It is readily seen that, in order to make sure that 
our model (9-10) includes the strong Allee effect in the limiting case $\tau=0$, the exponent $n$ must be larger than one; correspondingly, below we choose $n=2$. Thus, we arrive at the following equation for the population density of adults:

$$
\frac{d y(t)}{d t}=\alpha \frac{y^{2}(t-\tau)}{1+\beta y^{m}(t-\tau)} \exp (-\delta \tau)-D y(t) .
$$

For the sake of simplicity, we keep the adult mortality rate $D$ to be constant. Also, for convenience, in the rest of the paper we consider $A=\alpha \exp (-\delta \tau)$ as a fixed parameter. This will allow us to complete an exhaustive analysis of possible dynamical regimes. Note that fixing $A=$ const can be also considered as a particular ecological scenario in which the mortality of juveniles is assumed to be small, i.e. $\tau \delta<<1$, so that $A \approx \alpha$.

Finally, to reduce the number of parameters in the model we shall consider a dimensionless version of (11) with only three parameters $g, \tau$ and $m$ :

$$
\frac{d y(t)}{d t}=\frac{y^{2}(t-\tau)}{1+y^{m}(t-\tau)}-g y(t),
$$

where $g=D \beta^{1 / m} / A$ and the new delay is given by the previous delay multiplied by $A /\left(D \beta^{1 / m}\right)$.

Equation (12) must be complemented with the initial conditions, or "history", given by $y(t)=$ $\theta(t),-\tau<t<0$, where $\theta(t)$ is a non-negative function. In the numerical simulations, for the sake of simplicity, we consider the case where the population was initially in a steady state, so that $\theta(t)=y_{0}$ =const. Numerical simulations of (12) were performed using a standard explicit Euler scheme with a sufficiently small time step ( $h=0.0001)$ : the required accuracy was verified by reducing the time step and comparison of the results of simulations.

\section{Simulations and results}

\subsection{Dynamical attractors of the model.}

We start our investigation of the model by exploring the system attractors, which describe the population dynamics in the large-time limit.

The model may have up to three stationary states $y_{1}, y_{2}$ and $y_{3}$ (see Appendix A). The extinction state $y_{1}=0$ always exists and is always locally attractive for any $\tau \geq 0$. This signifies that the population will eventually go extinct if its density during time $\tau$ is low. In the case where the mortality $g$ is sufficiently small, there can be two extra stationary states: $y_{2}$ and $y_{3}\left(y_{2}<y_{3}\right)$. The intermediate state $y_{2}$ is always unstable, whereas the upper state $y_{3}$ (we shall call it the persistence stationary state) can be either stable or unstable depending on the magnitude of the delay. In the absence of delay, $y_{3}$ is always 
stable and we thus have a bi-stable model, which is equivalent to the classical population model with a strong Allee effect based on the cubic polynomial parametrization (cf. Lewis and Kareiva, 1993). For a supercritical delay $\tau$, the persistence stationary state $y_{3}$ may lose its stability. This occurs only for small and intermediate mortality rates $g$ (Appendix A), whereas for larger values of $g$ (in the case where $y_{3}$ still exists) this state remains stable for any delay $\tau$, thus it is absolutely stable.

The bifurcation portrait of the model in the $(g, \tau)$ plane is shown in Fig.1, which is constructed for $m=4$. The portrait is obtained from numerical simulation of (12) over a long time ( $t>2000)$ after removing the initial transient dynamics. Also, we use analytical results to plot the local bifurcation curves $H$ and $L$ (see Appendix A). Different colours and numbers of the domains correspond to different dynamical regimes. The vertical line $L$ is a saddle-node bifurcation curve: in domain 1 only the stationary state is the extinction $y_{1}=0$. In domain 2 , the system is bi-stable with locally stable states $y_{1}$ and $y_{3}$. Delay-induced destabilization of $y_{3}$ takes place on the Hopf bifurcation curve $H$, when entering domain 3 , and results into the appearance of a stable limit cycle around $y_{3}$. A further increase in $\tau$ results in complex sustained oscillations around $y_{3}$. The complexity of the oscillatory dynamics can be characterized by the number of consecutive local maxima of $y(t)$ through the oscillation period, which is depicted by various colours.

Fig.2 provides the cross section of Fig. 1 constructed for $g=0.15$ and shows the density of species at consecutive local maxima of oscillations. Two main types of bifurcations are observed in the delay destabilized model: (i) peak-adding bifurcations and (ii) period-doubling bifurcations. Examples of the two types of bifurcations are shown in Fig.3. In a peak-adding bifurcation a supplementary local maximum of $y(t)$ emerges (Fig.3a-b) and the period of oscillations is conserved at the moment when the bifurcation occurs . The main benchmark of this bifurcation is the sudden, discontinuous appearance or disappearance of new branches of local maxima of $y(t)$ in Fig.2. In the $y(t)-y(t-\tau)$ plane, a peak-adding bifurcation corresponds to a loop formation on the null-cline of the system shown in red (see Fig.S1 in Appendix B). On the other hand, a period doubling bifurcation is characterized by an increase of the period of oscillations by the factor of 2, which is shown in Fig.3cd. The corresponding $y(t)-y(t-\tau)$ plane diagrams are provided in Fig.S2 in Appendix B. The number of peaks in this bifurcation remains the same and a discontinuous appearance of new branches of local maxima of $y(t)$ is not observed.

An increase in the magnitude of the time lag $\tau$ results into a set of peak-adding bifurcations combined with period doubling bifurcations (see Fig.2) and eventually, the interplay between these two types of bifurcation leads to highly irregular oscillations of species density, without any apparent periodicity. This is observed for intermediate time delays. A thorough investigation of those patterns 
reveals that the dynamics are highly sensitive to initial conditions, which indicates that the irregular oscillations of species density are chaotic (see Fig.S3 in Appendix C): initially close trajectories will eventually diverge exponentially because the leading Lyapunov exponent is positive. In the $(g, \tau)$ diagram (Fig.1) the domain corresponding to chaotic regime is depicted in black. One can see that the delay-induced chaos in the system can be observed only within limited range of the other model parameter $g$. It is also worth noting that the boundaries of the domains corresponding to complex multi-periodic and chaotic regimes in the $(g, \tau)$ diagram appear to be simple, this is an effect of a coarse resolution. A finer resolution would reveal fractal boundaries between these domains, but research into the fractal structure of the $(g, \tau)$ diagram is a separate topic for investigation and should be done elsewhere.

Finally, we should emphasize that sustained oscillations occur only in the case where the initial species density is sufficiently high, otherwise, the population will eventually go extinct. In the infinite dimensional functional space given by $y(t)=y_{0}(t)$ for $-\tau<t<0$, the basin of attraction of the extinction state and persistence dynamics is rather complex. We will address this interesting issue in the next section.

\subsection{Long-term transient population dynamics.}

Extensive numerical simulation of the model shows that for large time interval- potentially up hundreds of generation times of the population, but of finite length- the patterns of population dynamics can be substantially different from those corresponding to the eventual system attractors.

We start our exploration of transient dynamics with the case that the persistence stationary state is absolutely stable, i.e. when the state $y_{3}$ cannot be destabilized for any $\tau>0$.Thus, there can be only two long term outcomes of dynamics, depending on the initial conditions: (i) the system settles on the persistence state $y_{3}$ or (ii) goes towards the extinction state $y_{1}=0$. Since the initial conditions of the system belong to the infinite dimensional functional space $y(t),-\tau<t<0$, we consider for the sake of simplicity that the $y(t)=y_{0}=$ const for $-\tau<t<0$. Our simulation shows that starting from either very low initial densities $y_{0}$, the trajectory quickly approaches the extinction state since the mortality rate is higher than reproduction rate. The same holds for very high initial densities $y_{0}$ since the mortality rate again is larger than the reproduction rate and the population densities quickly drop to low values with a further extinction. On the other hand, for values $y_{0}$ sufficiently close to $y_{3}$, the trajectory quickly settles on this state.

On the other hand, for some intermediate range of initial densities $y_{0}$ the population dynamics exhibit substantially different behaviour: the population size shows long term oscillations around the unstable state $y_{2}$. Interestingly, after a few initial fluctuations (see Fig. 4) the amplitude of oscillations 
becomes quasi constant over a long time period (up to 20-40 periods of oscillations) and suddenly the trajectory returns to one of the final stationary states. This pattern of dynamics can be considered as a transient limit cycle, where the trajectory initially approaches the cycle, remains on it for a while with constant amplitude and finally leaves the cycle. The duration of the transient oscillations depends on the time delay $\tau$ and the initial condition $y_{0}$. Fig.5 (left column) shows how $N$, the number of transient oscillations before the trajectory finally settles on the stable state, depends on $y_{0}$ and $\tau$. Note that when constructing Fig.5 we count only large supercritical oscillations and disregard relaxation oscillations, see the figure caption for details. From this figure, one can see that the dependence of $N$ on the magnitude of the delay is not monotonic: for small $\tau$ the system behaves as a classical bi-stable population model and the population size quickly approaches to one of the stable states, depending on the initial density $y_{0}$. For sufficiently large $\tau$, the population sizes also quickly approach the stationary states, but for some intermediate delay values we should expect the existence of very long-term transient limit cycles.

It is interesting to consider how the final destinations of the trajectories depend on $y_{0}$ and $\tau$, which is shown in the right column of Fig. 5. Dark blue corresponds to the eventual extinction of the population whereas red indicates that the system will be at the persistence state. Within the chosen range of the initial density $y_{0}$, and given parameter values, the delay-free model always predicts persistence of the population since we always have $y_{3}<y_{0}$, so adding a supercritical delay may cause the extinction of the population. The basins of attraction of $y_{1}$ and $y_{3}$ have a complex structure showing intermittency of red and blue stripes, thus a graduate increase in the magnitude of delay can potentially result in either extinction or rescue of the population.

We also investigated the dependence of the transients and long term asymptotic dynamics on the other model parameter $m$, which accounts for the strength of the Allee effect in the model. We found that an increase in $m$ leads to shrinking of the whole bifurcation diagram in the $(g, \tau)$ plane, but the main bifurcation structure remains qualitatively similar to the one shown in Fig.1. We also found long-term transient oscillations for $m=6,8,10$ similar to those observed in Fig.4. We can therefore conclude that the long-term transients in the model are robust to a variation of parameters in terms of $\tau$ and $m$ as well as the initial condition $y_{0}$.

Finally, long-term transient oscillations of the population size are not only observed in the case when the state $y_{3}$ is stable but also in the case, where $y_{3}$ loses its stability and the final attractor is a limit cycle or chaotic oscillations. Fig. 6 shows an example of a long-living transient population cycle around the unstable saddle state $y_{2}$. In Fig. 6A, the final attractor is the periodic limit cycle. Here the amplitude of the transient oscillations remains constant whereas the shape of the oscillations gradually 
changes and finally the trajectory quickly approaches the system attractor- the stable limit cycle. More complex transient oscillations occur in the case where the eventual attractor is chaotic, as is shown in Fig.6B. One can see that the shape of the transitional oscillations around $y_{2}$ is highly irregular, particularly concerning the population densities near the peaks; the drop of the population density to low values is still periodic, however. Analysis of time series of such transient dynamics can be a challenge since conclusions about the periodicity or chaoticity of the system will depend on the way the data points are chosen. The basins of attractions of the extinction and oscillatory states in the $\tau-y_{0}$ plane also show a high degree of complexity, thus small fluctuations in $\tau$ or $y_{0}$ can substantially change the long term fate of the population. We do not show these basins of attraction in the $\tau$ - $y_{0}$ plane here for the sake of brevity.

\section{Discussion}

In this paper, we have explored how the interplay between effects of maturation and the strong Allee effect can shape the population dynamics. Our motivation for this study was to build a minimal model that would account for some of the most common features of population ecology whilst remaining capable of simulating sufficiently complex dynamics including long-living transients and chaos that are thought to play an important role in ecology. Our model is conceptual because it omits unnecessary details, yet it is realistic in the sense that it overcomes the shortcomings of the logistic equation where individuals start reproducing immediately after they are born, which is biological nonsense.

Contemporary theoretical ecology relies heavily on predictions and inferences from mathematical modelling, and over the last several decades a great variety of models of various complexity have been developed ranging from very simple to very complicated. There has been considerable experience gained, and there is currently a good understanding as to what a "realistic" model should be (multi-species, multi-scale, hybrid etc.) to describe the population dynamics of given species in sufficient detail, although the limits of modelling complexity remain a controversial issue (Pascual 2005; Adamson and Morozov 2012; Petrovskii and Petrovskaya 2012; Morozov and Petrovskii 2013).

Ironically, the situation appears to be less clear at the other end of the spectrum, i.e. with simple conceptual models. Such models do not claim to have much predictive power or to embrace all the details, but are instead used to reveal some basic, fundamental properties of the ecological dynamics. Talking specifically about the population dynamics of a species with overlapping generations, the logistic equation used to be regarded as a minimum sensible model, and does indeed 
well describe the properties of some simple systems such as, for instance, a single-species algae population with one limiting resource (Brezonik 1993; Scheffer 1998; Yang 2011).

The logistic equation, however, predicts only very simple dynamics, such as monotonic convergence to a steady state, and this is clearly at odds with the complexity of the dynamics observed in natural populations where sustained oscillations are much more typical (Ranta et al., 1995; Cushing et al., 2003; Turchin, 2003; Sherratt and Smith, 2008). This is of course hardly surprising, as the logistic equation obviously leaves many things out of focus; in particular, it assumes that offspring start reproducing the moment they are born and are born immediately after they are conceived. The question arises as to how the logistic equation can be modified in some minimal way to keep the model as simple as possible yet make its properties nontrivial. One way to do this was suggested by Hutchinson in his seminal paper (Hutchinson 1948) where he showed that taking into account competition during gestation by including a time delay changes the model properties significantly, so that for a sufficiently long delay the population oscillates rather than converges.

Hutchinson’s paper (1948) was hugely influential, eventually resulting in a vast number of publications exploring the effects of time delays in population dynamics, e.g. see Cushing (1977), Gopalsamy (1992), Kuang (1993), Smith (2011) and the references therein. Yet despite this it can hardly be regarded as a sensible model - even as a conceptual model - for the following two reasons. First, like the logistic equation it still assumes that offspring start reproducing immediately after their birth. And second, the inclusion of the time delay into Hutchinson's equation is purely heuristic. Whilst it does not result in much ambiguity in the case of logistic-type growth, it immediately leads to a problem if the Allee effect is present. In this case, it is not clear where the time delay should be included, and its inclusion into different parts of the equation can have a very different effect (Jankovic and Petrovskii 2014). Correspondingly, our goal in this paper is to derive the simplest sensible model accounting for maturation time. This was done in Section 2. We should mention that an equation similar to our Eq.(12) but without the Allee effect, was first considered (but not derived) in the context of medical applications and is often referred to as the Mackey-Glass model (Mackey and Glass 1977). The Mackey-Glass model is known to have rich dynamics including periodic, multiperiodic and chaotic oscillations (Mackey and Glass 1977). In this paper, we have shown that the modification of the Mackey-Glass model that includes the strong Allee effect preserves the dynamical complexity of the original model but, additionally, is also capable of producing long-living transients (see Figs.4 and 6) and intermittent dynamics.

Note that in nonlinear models the evidence for regime shifts as a result of long living transients can be demonstrated mainly by numerical simulations. Thus, searching for long living transients in 
various specific systems is currently the main methodology to explore this complex phenomenon, with the goal to collect evidence of how widespread such dynamics are in population models. This is especially important taken into account the well-known property of structural sensitivity of biological models, where very close mathematical systems using different parameterisations of functions can predict completely different patterns of population dynamics (Fussmann and Blasius, 2005; Cordoleani et al., 2011; Adamson, and Morozov, 2012, 2014). Interestingly, long-term transients were obtained in another delay model using a different parameterisation of the Allee effect (i.e. based an exponential decay of the growth rate at large density y; see Liz and Ruiz-Herrera, 2015). Therefore, the existence of long living transients seems to be a robust property of this class of models. We mention here that, for an exhaustive comparison between the two models using different parameterisations, one should also investigate the dependence of the trajectories' final destination on the initial conditions and the maturation delay. Unfortunately, such dependence was not investigated by Liz and Ruiz-Herrera (2015). However, it has been done in this paper for our model (12) where the complex structure in $\left(y_{0}, \tau\right)$ parameter plane has been demonstrated (see Fig.5). Another interesting feature which we found in model (12) and, which needs to be verified in other similar models, is that long term transients occur even in the case where the final attractor is complex, for instance when it is chaotic (Fig.6B).

Based on the rich behaviour of our model, and taking also into account the clear and arguably ecologically sensible assumptions behind its derivation, we conclude that Eq.(11) (or Eq.(12) in dimensionless variables) would make a good candidate to replace the logistic equation and the Hutchinson equation as the "minimum conceptual model” of population dynamics. In particular, the existence of long-living transients (including chaotic transients) is one of the main properties that makes Eq.(12) interesting and relevant. Long-living transients are increasingly recognised as an important property of ecological dynamics (Hastings 2001, 2004) but the mechanisms resulting in them largely remain obscure. It is not even clear whether transients are a common property of natural ecosystems or whether it is an exotic phenomenon. In this paper, we have shown that long-living transients can arise from the interplay between the strong Allee effect (as determined by the sigmoidal shape of the per capita growth rate $r(y)$, see Eq.(10)) and the time delay. The presence of the time delay in the growth term in our model is not an assumption but a well-established fact: as is shown by the model derivation, it takes into account maturation which is a basic property of all biological species. We therefore conclude that, somewhat counter-intuitively, the existence of long-living transients is an inherent property of any species whose population growth rate is affected by a strong Allee effect. 


\section{References}

Adamson, M.W. and Morozov, A. Yu. 2013. When can we trust our model predictions? Unearthing structural sensitivity in biological systems. Proc. R. Soc. A, vol. 469, 20120500.

Adamson, M.W., and Morozov, A. Yu., 2014b. Bifurcation analysis of models with uncertain function specification: how should we proceed? Bull. Math. Biol. 76, 1218-1240.

Allen LJS (2007) An introduction to mathematical biology. Pearson Prentice Hall, Upper Saddle River, NJ.

Aiello WG, Freedman HI, 1990. A time-delay model of single-species growth with stagestructured. Math Biosci 101:139.

Allee, W.C., 1931. Animal Aggregations: A Study in General Sociology. University of Chicago Press, USA.

Becks, L., Hilker, F. M., Malchow, H., Jurgens, K., and Arndt, H. (2005) Experimental demonstration of chaos in a microbial food web. Nature 435, 1226-1229.

Begon, M., Townsend, C. R. Harper, J.L, 2005. Ecology: From Individuals to Ecosystems, 4th edn. London: Blackwell Publishing.

Berec, L., Angulo, E., Courchamp, F., 2007.Multiple Allee effects and population management. TREE 22, 185-191.

Beretta E, Kuang Y (1998) Global analyses in some delayed ratio-dependent predator-prey systems. Nonlinear Anal Theory Methods Appl 32(3):381-408

Bjornstad, O.N. and Grenfell, B.T. (2001) Noisy clockwork: time series analysis of population fluctuations in animals. Science 293, 638-643.

Boettiger C., Hastings A. 2012.Quantifying limits to detection of early warning for critical transitions. J. R. Soc. Interface9, 2527-2539.

Boukal D.S., Berec L. (2002) Single-species models of the Allee effect: extinction boundaries, sex ratios and mate encounters. J. Theor. Biol. 218, 375-394.

Brezonik P.L. (1993) Chemical Kinetics and Process Dynamics in Aquatic Systems. Boca Raton: CRC Press.

Cao Y. and GardT.C, Ultimate bounds and global asymptotic stability for differential delay equations, Rocky Mountain J. Math.25 (1995), 119-131.

Carpenter S. R (2003). Regime Shifts in Lake Ecosystems: Pattern and Variation. Oldendorf/Luhe, Germany: Ecology Institute. 199 p.

Cordoleani F., Nerini D., Gauduchon, M., Morozov A, Poggiale J.-C., 2011 Structural sensitivity of biological models revisited. J. Theor. Biol. 283, 82-91. 
Costantino, R. F., Desharnais, R. A., Cushing, J. M., Dennis, B., 1997. Chaotic dynamics in an insect population. Science 275, 389-391.

Courchamp, F., Clutton-Brock, T., Grenfell, B., 1999.Inverse density dependence and the Allee effect. TREE 14, 405-410.

Courchamp F, Berec J, Gascoigne J (2008). Allee effects in ecology and conservation. Oxford, New York, USA: Oxford University Press.

Cushing J M, Costantino R F, Dennis B, Desharnais R A , Henson S M, 2003. Chaos in ecology: experimental nonlinear dynamics. Amsterdam, Academic Press.

Cushing, J.M. Dennis, R. A. Desharnais, R. F. Costantino (1998). Moving toward an unstable equilibrium: saddle nodes in population systems. J. Anim. Ecol. 67, 298-306

Dennis, B., 1989. Allee effects: population growth, critical density, and the chance of extinction. Nat. Res. Model. 3, 481-538.

Ellner, S.P., Turchin, P., 1995. Chaos in a noisy world: new methods and evidence from time series analysis. American Naturalist 145, 343-375.

Fussmann GF, Blasius B. 2005 Community response to enrichment is highly sensitive to model structure. Biol. Lett. 1, 9-12.

Gopalsamy K. and Ladas, G. On the oscillation and asymptotic behavior of $\dot{N}(\mathrm{t})=N(\mathrm{t})\left[\mathrm{a}+\mathrm{b} N(\mathrm{t}-\tau)-\mathrm{c} N^{2}(\mathrm{t}-\tau)\right]$, Quart. Appl.Math. 48(1990), 433-440.

Gurney, W.S.C., Nisbet, R.M., Lawton, J.H. (1983). The systematic formulation of tractable single-species population models incorporating age structure. J Anim. Ecol. 52: 479-495.

Hansen T. F, Stenseth NC, Henttonen H, Tast J (1998) Interspecific and intraspecific competition as causes of direct and delayed density dependence in a fluctuating vole population. Proc National Acad Sci USA 96(3):986-991

Hastings A (1984) Delays in recruitment at different trophic levels: effects on stability. J Math Biol 21(1):35-44

Hastings, A. 2001.Transient dynamics and persistence of ecological systems. Ecol. Lett. 4, $215-220$.

Hastings, A. (2004). Transients: the key to long-term ecological understanding? Trends in Ecology and Evolution, 19:39-45.

Hutchinson GE (1948) Circular causal systems in ecology. Annals New York Acad Sci 50:221-246

Ims, R. A., J.-A.Henden, and S. T. Killengreen. 2008. Collapsing population cycles. Trends in Ecology and Evolution 23 (2): 79-86. 
Jankovic M., Petrovskii, S., 2014 Are time delays always destabilizing? Revisiting the role of time delays and the Allee effect. Theoretical Ecology 7, 335-349.

Kéfi S, Guttal V, Brock WA, Carpenter SR, et al. 2014.Early Warning Signals of Ecological Transitions: Methods for Spatial Patterns. PLoS ONE 9(3): e92097

Kuang, Y., 1993. Delay Differential Equations With Applications in Population Dynamics. Academic Press, New York.

Lai Y.C., Winslow, R.L. (1995). Geometric-properties of the chaotic saddle responsible for supertransients in spatiotemporal chaotic systems. Phys. Rev. Lett., 74: 5208-5211

Lewis, M.A., Kareiva, P., 1993. Allee dynamics and the spread of invading organisms. Theor. Popul. Biol. 43, 141-158.

Liz, E., Ruiz-Herrera, A. (2015). Delayed population models with Allee effects and exploitation. MBE 12 (1), 83-97.

Luckinbill, L.S., 1974. The effects of space and enrichment on a predator-prey system. Ecology 55, 1142-1147.

Maynard Smith J (1974) Models in ecology. Cambridge University Press, Cambridge.

May, R.M., 2001.Stability and Complexity in Model Ecosystems. Princeton University Press, Princeton

Merdan H, Duman O, Akin O., Celik C (2009) Allee effects on population dynamics in continuous (overlapping) case. Chaos, Solitons Fractals 39:1994-2001.

Morozov, A., and Petrovskii, S.V. (2013) Feeding on multiple sources: towards a universal parameterization of the functional response of a generalist predator allowing for switching. PLoS One 8(9), e74586.

Murdoch, W.W., Briggs, C.J., Nisbet, R.M., 2003. Consumer-Resource Dynamics. Princeton University Press, Princeton.

Nicholson, A.1954.An outline of the dynamics of animal populations.Aust. J. Zool. 2, 9-65.

Odum, E. P., Barrett, G. W. (2005).Fundamentals of ecology. Thompson Brooks/Cole, Belmont, CA.

Ranta E., Kaitala, V., Lindstrdm, J. and Linden, H. 1995. Synchrony in population dynamics.Proc. R. Soc. Lond. B 262: 113-118.

Rodrigues, L.A.D., Mistro, D.C., and Petrovskii, S. (2011) Pattern formation, long-term transients, and the Turing-Hopf bifurcation in a space- and time-discrete predator-prey system. Bull. Math. Biol. 73: 1812-1840. 
Ruan, S. (2006). Delay differential equations in single species dynamics, in Delay Differential Equations and Applications, ed. by O. Arino et al. (Springer, Berlin), 477-517.

Ruan S (2009).On nonlinear dynamics of predator-prey models with discrete delay. Math Model Nat Phenom 4:140-188

Scheffer M. (1998) Ecology of Shallow Lakes. London: Chapman and Hall.

Scheffer, M., Carpenter, S., Foley, J.A. Folke, C., Walkerk, B. 2001. Catastrophic shifts in ecosystems. Nature 413, 591-596.

Scheffer M, Bascompte J, Brock WA, Brovkin V, Carpenter SR, et al. (2009) Early-warning signals for critical transitions. Nature 461: 53-59.

Schreiber, S. 2003. Allee effects, chaotic transients, and unexpected extinctions, Theor. Pop. Biol. 64, 201-209.

Sen, M., Banerjee, M., Morozov, A. 2014. Stage-structured ratio-dependent predator-prey models revisited: When should the maturation lag result in systems' destabilization? Ecological Complexity19, 23-34.

Sherratt, JA, Smith, MJ, 2008. Periodic travelling waves in cyclic populations: field studies and reaction-diffusion models, R. Soc. Interface 5 (22), 483-505.

Stephens, P. A., Sutherland, W. J., 1999. Consequences of the Allee effect for behaviour, ecology and conservation. TREE 14, 401-405.

Takeuchi, Y. (1996). Global Dynamical Properties of Lotka-Volterra Systems. Singapore: World Scientific.

Tilman, D. May, R.M., Lehman, C.L., Nowak, M.A. (1994). Habitat destruction and the extinction debt. Nature 371, 65-66.

Turchin P. (2003) Complex Population Dynamics: a Theoretical/Empirical Synthesis. Princeton: Princeton University Press.

Widdicombe, C.E., Eloire, D., Harbour, D., Harris, R.P., Somerfield, P.J. (2010).Long-term phytoplankton community dynamics in the Western English Channel. J. Plankton Res., 32, 643-655.

Yang J., Rasa E., Tantayotai P., Scow K.M., Yuan H., Hristova K.R. (2011) Mathematical model of Chlorella minutissima UTEX2341 growth and lipid production under photoheterotrophic fermentation conditions. Bioresource Technology 102: 3077-3082.

Yoshida, T., Jones, L.E., Ellner, S.P., Fussmann, G.F. and Hairston, N.G., Jr. (2003). Rapid evolution drives ecological dynamics in a predator-prey system. Nature 424, 303-306. 


\section{Figure Captions}

1. Bifurcation diagram in the $(g, \tau)$ plane showing the attractors of the delay model constructed for $m=4$.The curve $L$ is a saddle-node bifurcation curve and $H$ is a Hopf bifurcation curve. The domains have the following meaning: Domain 1 - the only stable stationary state is $y_{1}=0$; Domain 2 bi-stability with the stable stationary states being $y_{1}=0$ and $y_{3}$; Domain 3- stable stationary state $y_{1}$ and a stable limit cycle around $y_{3}$; Domains 4, 5,6,7,8 - stable stationary state $y_{1}$ and periodic oscillations around $y_{3}$ with the number of local maxima in the period is 2, 4, 6, 8 and >8, respectively; Domain 9 stable state $y_{1}$ and chaotic oscillations around the unstable stationary state $y_{3}$.

2. Cross section of the diagram shown in Fig.1constructed for $g=0.15$. We show points of consecutive local maxima of $y(t)$ through oscillations. The two main types of bifurcations are illustrated in Fig.3.

3. Two main types of non-local bifurcations in the delay model. (a)-(b) peak-adding bifurcation: a supplementary local maximum emerges at point $B$. (c)-(d) period-doubling bifurcation: the period of oscillations increase by a factor of 2 with only small changes in the shape of the trajectory $y(t)$ (compare $A, B$ and $A^{\prime}, B^{\prime}$ ). The model parameters are $g=0.15, m=4$.

4. Long term transient oscillations in the delay model in the case where the final attractors are stationary states. The population size exhibits long term oscillations with quasi-constant amplitude around the unstable equilibrium $y_{2}$ before finally approaching either the persistence equilibrium (a) or extinction equilibrium (b). The model parameters are $g=0.15, m=4$.

5. Dependence of transient oscillations on the initial density $y_{0}$ and the value of delay $\tau$.

Left hand column: in each diagram the number $N$ of large amplitude transient oscillations is shown. We count only those oscillations with $y_{\min }(t)<0.9 y_{3}$ and $y_{\max }(t)<1.1 y_{1}$, thus excluding small amplitude relaxation oscillations around the stationary states. The diagrams (a)-(c) are constructed for $g=0.55, g=0.5, g=0.45$, respectively. Right hand column: basins of attractors of $y_{1}$ (extinction) and $y_{3}$ (persistence) states are shown, depicted by red and dark blue, respectively. The diagrams (d)-(f) are constructed for $g=0.55, g=0.5, g=0.45$, respectively. The other model parameter is $m=4$.

6. Long term transient oscillations in the model in the case where the persistence attractor is non-stationary. (a) The final attractor is a limit cycle. The model parameters are $m=8 ; g=0.44 ; \tau=70$; $y_{0}=1.55$. (b) The final attractor is chaotic. The model parameters are $m=6 ; g=0.45 ; \tau=50 ; y_{0}=1.8$. 